\title{
Experiencia etnoeducativa en la institución educativa rural bilingüe artesanal kamëntŝá, Valle del Sibundoy, Colombia
}

\author{
Omar Jacanamejoy-Juajibioy \\ Universidad de Narińo, Pasto-Colombia \\ omar2j_@udenar.edu.co \\ Lilia Concepción Juajibioy Jamioy \\ Institución Educativa Rural Bilingüe Artesanal Kamëntŝá, Sibundoy-Colombia \\ liliacjj@gmail.com \\ Hilbert Blanco-Álvarez \\ Universidad de Narińo, Pasto-Colombia \\ hilbla@udenar.edu.co
}

Recibido: 9 - febrero - 2018 / Aceptado: 31 - julio - 2018

\section{Resumen}

Este artículo presenta la experiencia etnoeducativa de la Institución Educativa Rural Bilingüe Artesanal Kamëntŝá ubicada en el Valle de Sibundoy, Putumayo, Colombia. Se inicia presentando los elementos constituyentes del currículo de la institución, luego se presentan las dificultades en la implementación de una educación que tiene en cuenta los elementos culturales de la comunidad indígena Kamëntŝá, posteriormente se describen algunos aprendizajes obtenidos en un proceso de más de 20 años. Finalmente, se expone la experiencia de la enseñanza de las matemáticas escolares y la problemática que se presenta al tratar de estudiar los elementos etnomatemáticos de la cultura.

Palabras clave: etnoeducación, currículo cultural, etnomatemática. 


\begin{abstract}
This article presents the ethno-educational experience of the Bilingüe Artisanal Kamëntŝá Rural Educational Institution located in the Sibundoy Valley, PutumayoColombia. In the first part, the constituent elements of the institution's curriculum are exposed, then the difficulties in the implementation of an education that takes into account the cultural elements of the Kamëntŝa indigenous community are presented, later some learning obtained in a process of more than 20 years. Finally, the experience of teaching school mathematics and the problems that arise when trying to study the ethnomathematical elements of culture are exposed.
\end{abstract}

Keywords: ethnoeducation, cultural curriculum, ethnomathematics 


\section{Introducción}

$7 \mathrm{n}$ el sur occidente colombiano, en el departamento de Putumayo, se en$\checkmark$ cuentra ubicado el territorio del Valle de Sibundoy, y en él, el municipio que lleva su mismo nombre, Sibundoy. Municipio donde habitan las comunidades indígenas Kamëntŝá e Inga, además de un gran número de personas no indígenas o llamados colonos. Cuenta con 5 instituciones educativas: la Institución Educativa Champagnat de Sibundoy, Escuela Normal Superior del Putumayo, Colegio Fray Bartolomé de Igualada, Colegio Seminario Misional de Sibundoy y la Institución Educativa Rural Bilingüe Artesanal Kamëntŝá. Esta última, perteneciente a la comunidad Kamëntŝá y se ubica en la zona rural, en la vereda Las Cochas, que corresponde a la parte plana del municipio. Esta institución se considera patrimonio de la comunidad, pues en ella se trabaja el fortalecimiento de los valores y prácticas culturales de la comunidad Kamëntsáá, a través de los diferentes espacios de vivencia y aprendizaje; y de igual forma, se trabaja lo que es la parte occidental, es decir, una educación integral bajo las leyes establecidas por el Ministerio de Educación Nacional de Colombia (MEN).

Durante las décadas de los 70 y 80 como se menciona en Patińo (2004) se dieron las grandes luchas de líderes indígenas preocupados por la conservación de su territorio, sus costumbres, su lengua y el futuro venidero de las nuevas generaciones. Hasta ese entonces no se tenía en cuenta a las comunidades indígenas como sujetos de derecho, sin embargo, algunas comunidades ya tenían su educación propia basada en sus valores y tradiciones culturales. En ese sentido, gracias a la Constitución de 1991 y a leyes posteriores como la Ley General de Educación de 1994, se obtiene bajo fundamentos legales, garantías educativas para las comunidades campesinas, afrocolombianas e indígenas, de acuerdo los principios, cosmovisión y necesidades de cada pueblo.

En Colombia, a pesar de caracterizarse por tener su diversidad cultural, en la mayoría de las instituciones educativas, el aprendizaje y la enseñanza de una determinada asignatura, en especial de matemáticas, se trabaja con el modelo tradicional, desde un enfoque monocultural que no aprecia el pensamiento matemático de las culturas distintas a la occidental que, por el contrario, contribuye a la pérdida del interés por aprender y rescatar el pensamiento y prácticas ancestrales de las comunidades indígenas y afrodescendientes (Peña-Rincón \& Blanco-Álvarez, 2015).

La institución educativa rural bilingüe artesanal Kamëntŝá, en materia etnoeducativa, se ha convertido en el epicentro más importante del Municipio del Valle de Sibundoy, con sus actividades pedagógicas y con sus actividades relacionadas con la cultura. Las artesanías, la lengua materna, la chagra, el territorio y otros elementos integradores del currículo, generan una dinámica pedagógica alrededor de las vivencias y quehaceres de los indígenas Kamëntŝá, con el propósito de conservar y rescatar los valores culturales legados por sus antepasados. En la figura 1, se presenta la institución y algunas de las actividades. 


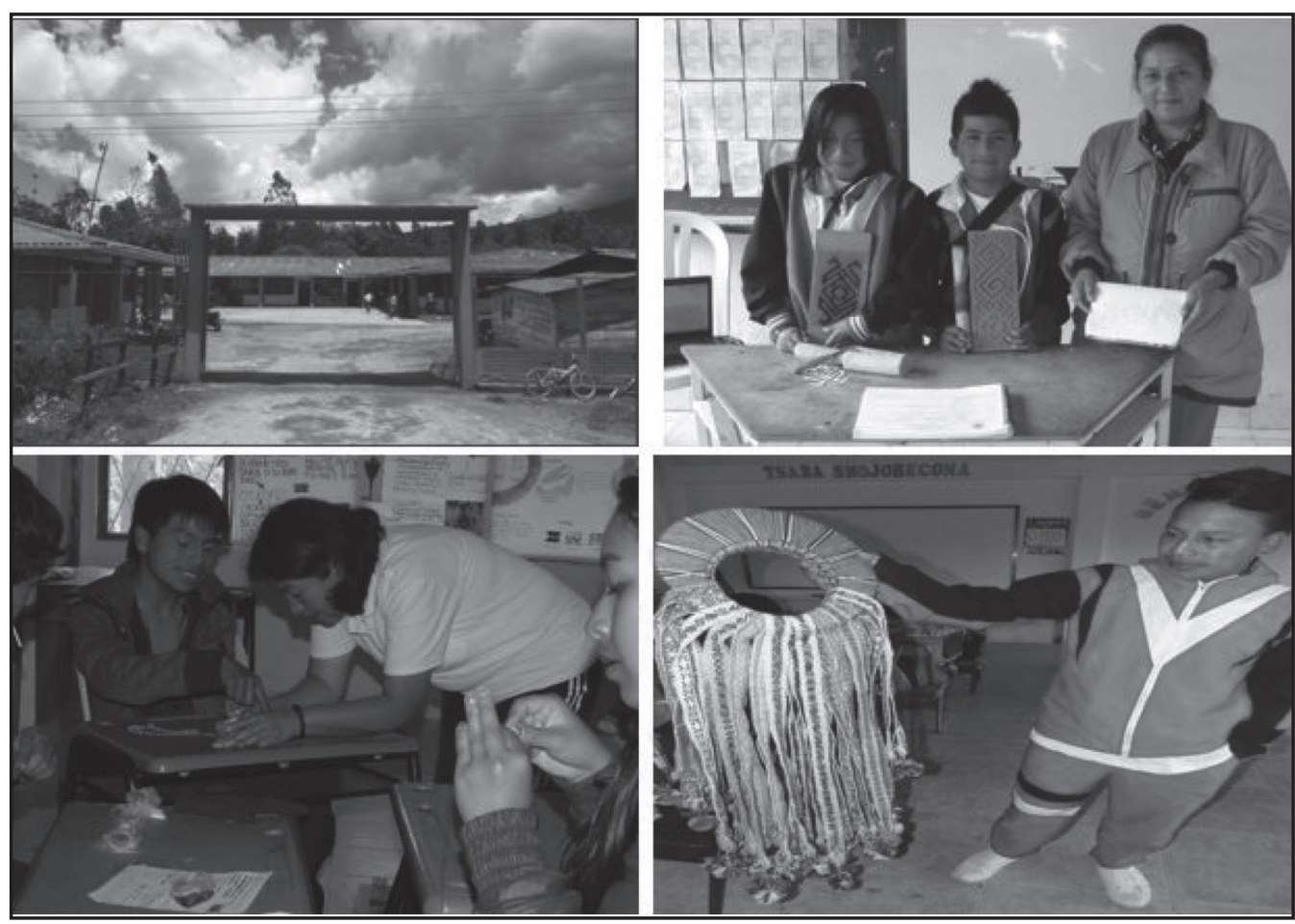

Figura 1. Institución educativa rural bilingüe artesanal Kamëntŝá y algunas de sus actividades.

Fuente: Propia

En lo que sigue, se presentarán los aspectos curriculares de esta institución; dificultades y aprendizajes obtenidos en la implementación de su currículo etnoeducativo y elementos etnomatemáticos presentes en él.

\section{Caracterización del currículo etnoeducativo de la institución educativa rural bilingüe artesanal Kamëntŝá}

En el marco de la ley 115 de 1994 o Ley General de Educación, en el capítulo 3, que garantiza una educación para grupos étnicos, en particular para comunidades indígenas que posean una cultura, una lengua, unas tradiciones y una jurisdicción propia y autóctona; se fundamenta el proyecto educativo del Colegio Bilingüe Artesanal Kamëntŝá perteneciente a la comunidad Kamëntŝá Biyá de Sibundoy, Putumayo, como una propuesta dinámica, en constante construcción y desarrollo colectivo de una educación intercultural bilingüe, que corresponda a la visión del hombre y sociedad, que el pueblo desea construir a partir de su propia cosmovisión, con la dimensión de interculturalidad dadas las situaciones vivenciales de espacio-tiempo con la proximidad hacia el siglo XXI.

Este proyecto fue creado por iniciativa de algunos miembros, líderes de la comunidad Kamëntŝá, con el único deseo de dar continuidad al proceso de formación 
con pensamiento indígena que se venía recibiendo desde el hogar infantil Basetemëngbe Yebna ${ }^{1}$. El propósito de este proyecto educativo es brindar una formación integral a los estudiantes indígenas con la colaboración y participación activa de todos los miembros que conforman la comunidad educativa, la autoridad tradicional, estamentos gubernamentales, no gubernamentales teniendo como bases procesos administrativos y pedagógicos mediante una formación dinámica con miras a capacitar a una persona de bien hacia los demás y hacia la autonomía, permitiéndole determinar sus propias características personales.

En ese sentido, adaptando la definición de currículo propuesta por el Ministerio de Educación Nacional de Colombia, en la cual establece que es el conjunto de los criterios, planes de estudios, programas, metodologías y procesos que contribuyen a la formación integral y a la construcción de la identidad cultural nacional, regional y local incluyendo también los recursos humanos, académicos y físicos para poner en práctica las políticas y llevar a cabo el proyecto educativo institucional; se presentan algunos aspectos del currículo del Colegio Bilingüe Artesanal Kamëntŝá, plan de estudio; programas adicionales, y metodologías en las que se plantea la creación de ambientes de aprendizaje que favorezcan el desarrollo de los educandos de esta institución.

De conformidad con el artículo 79 de la Ley 115 de 1994 (MEN, 1994a) y el artículo 38 del Decreto 1860 de 1994 (MEN, 1994b), se considera el Plan de Estudios como el esquema estructurado de las áreas obligatorias y fundamentales, y de las áreas optativas con sus respectivas asignaturas y proyectos pedagógicos. En ese sentido, el plan de estudios se rige por los criterios establecidos en su horizonte institucional y las normas estipuladas en la ley 115 de 1994 (artículo 23 y 31), donde se determinan las áreas obligatorias, de la cultura Kamëntŝá, optativas, las asignaturas obligatorias y demás acciones de carácter académico y pedagógico, así:

- Ciencias Naturales y Educación Ambiental

- Ciencias Sociales

- Educación Artística

- Educación Ética y Valores Humanos

- Educación Física, Recreación y Deportes

- Educación Religiosa

- Área de Humanidades e Inglés

- Matemáticas

- Tecnología e Informática

Las cuales, se establecen como áreas obligatorias y fundamentales del conocimiento, estipuladas en la Ley General de Educación (artículo 23), para el logro

1 La educación indígena para el pueblo Kamëntŝá inició con la conformación del hogar infantil Basetemëngbe Yebna (La casa de los niños), donde se practica valores culturales y se enseña la lengua materna. 
de los objetivos de la educación básica, que exige el Ministerio de Educación Nacional. Para la educación media, igualmente, como se establece en el artículo 31, serán obligatorias y fundamentales, las mismas áreas con un nivel de profundización avanzado. Además de ciencias políticas y filosofía. En el caso de ciencias políticas, el Colegio Bilingüe trabaja desde la parte tradicional Territorio y política indígena, que ya veremos más adelante.

Por otra parte, esta misma Ley (artículo 77) brinda autonomía a las instituciones para organizar áreas optativas dentro de las áreas establecidas conforme con el proyecto educativo institucional, adaptando algunas áreas a las necesidades y características regionales; métodos de enseñanza y organización de actividades formativas, culturales y deportivas, dentro de los lineamientos que establezca el Ministerio de Educación Nacional, con la finalidad de conservar y rescatar los usos y costumbres de la comunidad Kamëntŝá. De ese modo, se organizan las áreas tradicionales de la cultura así:

\section{Áreas de la Cultura Indígena Kamëntŝá \\ - Lengua Materna Kamëntŝá}

La cual se considera única en el mundo, proveniente de la familia Malayo-Polinesia, según el Dr. Manuel José Casas Manrique, como se menciona (Colegio Bilingüe Artesanal, 2015), es la lengua propia y autóctona de la comunidad Kamëntŝá, término atribuido como nombre de la comunidad y también hace referencia a la lengua propia $(\mathrm{Ka}=$ =mismo, mën= ser o estar, $\mathrm{ts}=$ lugar, á=persona $)$ que contextualmente significa, persona que pertenece al grupo étnico y hablante de un mismo idioma, el Kamëntŝá. Es una de las preocupaciones más grandes por los docentes y en general por la toda la comunidad, lo manifestaron en la entrevista realizada como veremos más adelante, pues cuando llegaron los españoles las familias Kamëntŝá tenían su educación a través de valores autóctonos, tenían una metodología natural para transmitir las costumbres y tradiciones que les legaron sus antepasados a las nuevas generaciones, utilizando como instrumento principal la tradición oral, la comunicación, la lengua materna o a través de las diferentes actividades del diario vivir, como el cuidado de la tierra, las artesanías, las mingas, y el compartir en familia alrededor del shinyak, ${ }^{2}$ donde los abuelos y padres contaban experiencias, cuentos y mitos, mientras los niños ayudaban en tareas de la casa, como desgranar maíz, frijol y otras actividades que poco a poco iban aprendiendo y cada día desarrollando habilidades y conocimientos de su propia cultura y formándose como hombre o mujer Kamëntŝá (Lilia Juajibioy, comunicación personal, 13 de julio de 2017). Estas tradiciones, en especial la lengua materna, se vinieron perdiendo con el paso del tiempo, tras la

2 Fogón donde preparan los alimentos. Antiguamente los mayores, solían reunirse a compartir experiencias, cuentos y mitos a los niños de la casa, después de haber consumido sus alimentos. 
llegada de los españoles, diferentes factores como la tecnología, la religión, la imposición de las costumbres externas y formas de pensamiento, incluso el mismo idioma español, fueron la causa del desinterés por aprender y cultivar este idioma.

Para fortalecer la lengua materna el Colegio Bilingüe, cuenta con el área de Kamëntŝá, como una asignatura más que se dicta en todos los grados, desde el más bajo hasta el superior. A través de la educación bilingüe se pretende cultivar las cuatro habilidades comunicativas: hablar, escuchar, leer y escribir en Kamëntŝá, mediante la relación interpersonal, la recopilación de cuentos, mitos y leyendas, tradiciones, a través de la investigación participativa de los estudiantes, docentes, padres de familia y comunidad, la práctica de valores, normas, costumbres y actitudes de la cultura con la convivencia mutua. Es decir, además de estar presente en el currículo, la lengua como una materia, también se la incluye en las clases de las otras asignaturas obligatorias y fundamentales utilizando palabras usuales, por ejemplo: el saludo, pedir permiso para ir al baño, pedir la palabra, etc., estas y otras situaciones que se presentan en el ambiente escolar ayudan al fortalecimiento y apropiación de la lengua Kamëntŝá. Con el mismo fin, en los diferentes espacios físicos, el colegio cuenta con un tipo de "señalización" de objetos (plantas medicinales, árboles, entre otros) y lugares comunes (restaurante escolar, senderos ecológicos, baños, entre otros) en lengua materna y su traducción al español, que sirve tanto para que los estudiantes se familiaricen con el entorno y de cierto modo aprendan la lengua, como también para las personas externas que visitan el colegio indígena.

$\mathrm{Al}$ igual que la lengua materna, el currículo etnoeducativo de la institución integra el área de artesanías con sus 4 modalidades, así:

- Tallado en madera (solo en secundaria)

- Tejido en orlón

- Tejido en fibra

- Tejido en chaquira

Las cuales se han venido transmitiendo de generación en generación, siendo los quehaceres y de cierto modo fuentes de ingreso para el sostenimiento de las familias Kamëntŝá. Con estas áreas propias de la cultura, el estudiante cada año adquiere nuevas habilidades y las perfecciona en la parte técnica, es decir, en la elaboración del producto artesanal como tal. Además, se promueve la investigación, pues los estudiantes para realizar un producto artesanal (tŝombiach ${ }^{3}$, máscara tradicional, sheknaj ${ }^{4}$, pulseras, collares, entre otros), primero consultan a los abuelos sabedores la importancia del objeto para la cultura, la historia y también la simbología que se plasma en la mayoría de ellos.

3 Faja tradicional Kamëntŝá elaborada en orlón que utilizan las mujeres en su vientre como traje autóctono.

4 Mochila tradicional Kamëntŝá elaborada con fibra, se utiliza para guardar la comida que se brinda en fiestas tradicionales. 
Otra área de la cultura, que se articula con las ciencias naturales y la educación ambiental y hace parte del currículo etnoeducativo de la institución es la Chagra y Medicina Tradicional. Aquí se enseñan los saberes tradicionales relacionados con el cuidado y trabajo de bëngbe tsbatsanamamá "nuestra madre tierra"; la siembra de los diferentes productos de la región; las fases de la Luna que se tienen en cuenta para cada actividad en el campo; la importancia y utilidad de las plantas medicinales para el Kamëntŝá. Se organizan mingas ${ }^{5}$ con los estudiantes y con la comunidad educativa, con el fin de cultivar la variedad de plantas (aromáticas, medicinales, hortalizas, etc.) existentes en el territorio y priorizando las que se encuentran en vía de extinción, que son utilizados para atender algunas de las necesidades de la institución como para el restaurante escolar o para el compartir ${ }^{6}$ comunitario cuando se realizan este tipo de actividades. Estos y otros saberes son compartidos y orientados por personas sabedoras: mamitas, parteras y taitas curanderos o Tatŝembwas en lengua materna, de la misma comunidad, que también hacen parte del proceso de formación integral de los estudiantes de esta institución.

$\mathrm{Al}$ igual que la lengua materna, las artesanías, y la chagra, el territorio hace parte del currículo etnoeducativo de la institución como área Territorio y política indígena. El territorio se considera como el origen de la vida. La tierra constituye el espacio de origen de la vida, el espacio de origen de la gente, las plantas, los animales, el agua, el viento, el día y la noche, es el espacio sagrado donde debe manifestarse el respeto y mantenimiento del equilibrio en el mundo natural. Para los Kamëntsá el territorio es un espacio vital, sin la tierra no puede haber existencia. Dentro de la historia están ligados los diversos procesos de colonización, desplazamiento, invasiones, recuperación, legalización, lo cual ha marcado profundamente a la comunidad.

Esta asignatura se trabaja únicamente en los grados 10 y 11 (últimos grados de la educación secundaria en Colombia), se abordan temas como: consulta previa y la historia de cómo los grandes líderes han luchado por el territorio que en estos tiempos aún tenemos, para que los estudiantes sepan de dónde vienen, su origen, fortalezcan su identidad, valoren el proceso de lucha y resistencia y así mismo sigan los pasos de esos grandes líderes, para no dejar perder el legado ancestral y brindar un mejor futuro para las generaciones venideras.

Por otro lado, el indígena Kamëntŝá como todo ser humano expresa sus conocimientos y sentimientos de alegría, de tristeza a través de la música y la danza que se caracteriza siempre por el acompañamiento del bombo y de la flauta, con sus tonos tradicionales los cuales fueron utilizados en las diferentes festividades de la comunidad. En ese sentido, la institución tiene el área de Música y Folclore como parte del currículo etnoeducativo donde se trabaja la música y danza autóctonas de los Kamëntŝá, como el bambuco, las diferentes melodías utilizadas para una ocasión 
especial y algunas danzas, como la de la siembra, la ofrenda, entre otras; incluyendo también la práctica de la lengua materna. Igualmente se trabaja la música latinoamericana, ya que, por ser un país andino, la influencia de las culturas cercanas es bastante alta. Espacios como estos, generan habilidades en ejecución de instrumentos tradicionales y andinos en los jóvenes Kamëntŝá, dominio de la lengua materna, e interés por investigar las tradiciones antiguas que practicaban los mayores antes de la colonización.

La Tabla 1 muestra, en síntesis, las áreas que se trabajan dentro del currículo, propuesto en el Proyecto Educativo Institucional (PEI) del Colegio bilingüe: las asignaturas obligatorias y fundamentales establecidas por el Ministerio de Educación Nacional de Colombia y las tradicionales pertenecientes a la cultura Kamëntŝá.

Tabla 1. Asignaturas del Currículo

\begin{tabular}{|c|c|c|}
\hline \multirow[t]{2}{*}{ Áreas de la Cultura } & Artesanías & $\begin{array}{ll}\text { - } & \text { Tallado en madera } \\
\text { - } & \text { Tejido en fibra } \\
\text { - } & \text { Tejido en lana orlón } \\
\text { - } & \text { Tejido en chaquira }\end{array}$ \\
\hline & $\begin{array}{ll}\text { - } & \text { Chagra y me } \\
\text { - } & \text { Lengua Kam } \\
\text { - } & \text { Territorio y }\end{array}$ & $\begin{array}{l}\text { radicional } \\
\text { ndígena }\end{array}$ \\
\hline $\begin{array}{l}\text { Áreas obligatorias y } \\
\text { fundamentales }\end{array}$ & $\begin{array}{ll}\text { - } & \text { Ciencias Na } \\
\text { - } & \text { Ciencias Soc } \\
\text { - } & \text { Educación A } \\
\text { - } & \text { Educación É } \\
\text { - } & \text { Educación F } \\
\text { - } & \text { Área de Hun } \\
\text { - } & \text { Matemáticas } \\
\text { - } & \text { Tecnología e }\end{array}$ & $\begin{array}{l}\text { Educación Ambiental } \\
\text { lores Humanos } \\
\text { creación y Deportes } \\
\text { es (Lengua Castellana e Inglés) } \\
\text { ática }\end{array}$ \\
\hline
\end{tabular}

Fuente: Propia

\section{Metodologías de trabajo}

La I.E. Bilingüe adopta proyectos pedagógicos desarrollados como un conjunto de actividades programadas para alcanzar objetivos, son una forma de generar y aplicar conocimientos en unidades de contenido, de acuerdo con las necesidades reales, por temas, núcleos, problemas, casos, etc. En forma personalizada y por grupos, haciendo un aprendizaje dinámico, creativo y útil para la vida. La actividad lúdica se constituye aquí en una herramienta de aprendizaje de ciencia y tecnología. 
Los proyectos pedagógicos facilitan los aprendizajes significativos de todas las disciplinas, en diversos grados y niveles y propician la aplicación de pedagogías activas y pedagogías propias de los grupos étnicos, procesos de integración de conocimiento y su aplicación a la vida diaria" (Peinado y Rodríguez, 2007, 67).

En ese sentido, esta institución ofrece proyectos de cultura, lectura, deporte, bienestar y religión con objetivos amplios que convergen hacia el fortalecimiento de la identidad cultural. De igual manera la institución con base en la ley 115, establece la obligatoriedad de Proyectos pedagógicos que contribuyan a la formación integral del educando, los organiza de la siguiente manera:

Proyectos obligatorios ley 115: Aprovechamiento del tiempo libre, el fomento de las diversas culturas principalmente la cultura Kamëntŝá, PRAE (educación ambiental, protección del ambiente, ecología y prevención de los recursos naturales), valores humanos (educación para la justicia la paz, la democracia, la solidaridad, confraternidad, cooperativismo), educación sexual, prevención de desastres y atención de emergencias, servicio social estudiantil, constitución nacional, civismo y urbanidad (PEI Colegio Bilingüe, 2015).

Por otra parte, al ser una institución enfocada al fortalecimiento de la identidad cultural y al pertenecer al gobierno tradicional de la comunidad Kamëntŝá, se celebran las fiestas tradicionales, donde se comparten distintos saberes que los mayores han venido transmitiendo de generación en generación. Por ejemplo: el wakjnayté "día de las ánimas" que es una ofrenda a los seres que han trascendido a lugares sagrados, y a la vez considerado como una antesala a la fiesta grande, la fiesta del perdón (Bëtsknaté), donde los estudiantes salen a relucir sus trabajos realizados en años anteriores en las cuatro modalidades de artesanías: coronas, fajas, collares, jigras y demás elementos que conforman el atuendo tradicional. Estos y demás espacios se desarrollan dentro y fuera de la institución, los cuales cumplen con su horizonte y filosofía establecidos en su proyecto educativo institucional.

\section{Personal docente}

En referencia al personal de planta docente, la institución bilingüe está orientada por educadores pertenecientes a la comunidad Kamëntŝá Biyá; personas preparadas desde la academia occidental en sus diferentes ramas del conocimiento. De igual manera, en un sentido profundo de pertenencia; conocedores, sabedores del pensamiento, lengua, valores y tradiciones de su cultura. Con el fin de transmitir y vivenciar a la niñez y juventud Kamëntŝá tanto los conocimientos convencionales que la academia establece como obligatorios y fundamentales, como el legado que dejaron sus abuelos mayores. Es decir, al ser una institución creada por la misma comunidad indígena, tanto docentes, estudiantes, padres de familia y en general la comunidad educativa se orientan en los principios filosóficos propios de la comunidad Kamëntŝá "unidad, fraternidad y lucha por los valores de la cultura", en un mundo cambiante. 
En síntesis, por lo señalado en el Capítulo 3 de la Ley General de Educación la Institución Educativa Bilingüe Artesanal Kamëntŝá es autónoma y responsable de la formación de los niños, niñas y jóvenes de la etnia Kamëntŝá mediante la transmisión y conservación de los valores ancestrales tales como la lengua, las costumbres, las artes, el tejido, el tallado, la cestería, la tintura natural, el tejido en chaquira, la elaboración de instrumentos musicales, entre otros, que transversalizan las diversas áreas pedagógicas establecidas por el Ministerio de Educación Nacional.

\section{Dificultades en la implementación de un currículo etnoeducativo en la institución}

Como se mencionó anteriormente, esta institución tiene como horizonte el fortalecimiento de la identidad cultural, particularmente la lengua materna: "El Kamëntŝâ"; a través de las prácticas escolares en los diferentes espacios físicos y de convivencia que ofrece la institución. En ese sentido, la comunidad educativa como principal apoyo de los estudiantes tiene la misión de contribuir al fortalecimiento diario de la lengua. Así mismo, por parte de los docentes, es una gran responsabilidad hacer seguimiento paralelo de la asignatura a cargo y el uso del idioma tradicional en sus determinados contextos.

La comunidad estudiantil de la institución bilingüe en su mayoría son indígenas, pertenecientes a la comunidad Kamëntŝá, algunos pocos de la comunidad Inga y el resto colonos (no indígenas). Varios jóvenes y padres de familia de la comunidad Kamëntŝá no ingresan a este centro educativo porque le restan importancia a cultivar los valores de la etnia, como son la lengua materna, las artesanías, el folclor y otras manifestaciones.

La propuesta de implementar áreas de la cultura que favorezcan el fortalecimiento de los valores y tradiciones culturales ha sido un proceso difícil, ya que en instituciones convencionales únicamente enfocan los conocimientos direccionados hacia las pruebas Saber (Pruebas nacionales estandarizadas realizadas por el Ministerio de Educación Nacional en Colombia). En cambio, en esta institución como se establece en su horizonte institucional, su gran interés es conservar la cultura. Sin embargo, el Ministerio de Educación no tiene en cuenta a las instituciones indígenas al momento de evaluar a los estudiantes en las pruebas Saber, por lo que han tenido que estructurar de la mejor forma su currículo para trabajar las áreas obligatorias y fundamentales que asigna el Ministerio y a la vez enfocar a sus alumnos al fortalecimiento de los saberes propios de su cultura. En consecuencia, muchos padres de familia no están conformes con la forma de distribuir las asignaturas y consideran que la educación en esta institución debería ser 100\% enfocada en la cultura.

Para determinar las dificultades que presenta la institución al implementar su currículo etnoeducativo, se entrevistó a tres docentes del área de artesanías. Se encontró que en su mayoría coinciden con las dificultades que se han venido presentando durante muchos ańos de vida escolar. 
Primera dificultad: los docentes entrevistados señalaron que la principal dificultad es la lengua materna, esto por muchas razones, una es porque no todos los estudiantes son indígenas, al ser una institución pública, no se niega el derecho, a ningún niño o niña, a ingresar a esta institución; dos, no todos los estudiantes tienen la disposición e interés por aprender y rescatar los elementos de cultura Kamëntŝá. En general, los niños y jóvenes de la comunidad Kamëntŝá, pese a los programas, espacios que ofrece la autoridad tradicional y los entes que contribuyen al fortalecimiento de los saberes culturales, son pocos en asistir debido al bajo interés y a la vergüenza por pertenecer a una comunidad indígena. $Y$ tres, por el proceso de aculturización, en un mundo cambiante existen padres de familia jóvenes no hablantes de la lengua materna, por ende, sus hijos, desde sus inicios de vida no reciben la semilla de fortalecimiento hacia su propia identidad como lo señala la docente Clara Juajibioy "Los niños vienen o sea cada vez [bueno] ya no se habla la lengua en las familias, en las parejas jóvenes y entonces viven como con serias dificultades en el uso de la lengua" (C. Juajibioy, comunicación personal, 13 de julio de 2017) y a su vez el docente Miguel Mutumbajoy menciona:

La mayor parte de los estudiantes no entienden la lengua materna, es una dificultad y un limitante, pero sin embargo uno ha hecho de que desde al área que esté encargado, trato de comunicar o expresarme con algunas palabras en la lengua materna, por ejemplo, les dijo: mochanjwabem, que significa vamos a escribir, o sea uno trata de alguna forma de tener en cuenta la lengua en cualquier clase o espacio dentro de la institución (M. Mutumbajoy, comunicación personal, 13 Julio 2017).

Segunda dificultad: una dificultad que hoy en día es muy común en términos de aprendizaje por parte de los estudiantes es la distracción causada por el mal uso de la tecnología. Los docentes expresaron que muchos estudiantes no muestran interés por aprender tanto los conocimientos convencionales que se trabajan normalmente en una institución educativa, como los valores, y costumbres de la cultura. Frente a esto la docente Clara Juajibioy manifiesta que:

Los jóvenes cada vez [o sea] salen más distractores ¿̨no?, por ejemplo, están casi la mayoría con el celular por acá (señala los pasillos donde la señal de internet es fuerte) desde por la mañana, desde que llegan se ubican donde les llegue más el internet y entonces prefieren estar más conectados con eso [hmm] bueno estar chateando [hm bueno y todo eso], y entonces vienen con esa distracción como tan difícil de cambiar ¿no? Y por esa razón no prestan atención por aprender bëngbe waman soy ${ }^{7}$ y en general ¿̨no? Lo que se trabaja en los colegios normales” (C. Juajibioy, comunicación personal, 13 de julio de 2017).

Tercera dificultad: la falta de recursos económicos necesarios para la creación de espacios pedagógicos adecuados para el desarrollo de las actividades de artesanías. Como se mencionó anteriormente, en la institución se desarrollan las 4 modalidades de artesanías, 
como son, tallado en madera, tejido en orlón, tejido en chaquira, y tejido en fibra. Frente a esto, la institución no cuenta con ambientes apropiados ${ }^{8}$ para el desarrollo de cada una de las modalidades, sin embargo, no es un limitante para cumplir a cabalidad los objetivos de fortalecer los saberes ancestrales como manifiesta la docente Clara Juajibioy:

(...) otra de facultad sería pues, o sea tener todos los recursos como necesarios, los espacios pedagógicos adecuados que se pueda [digamos] como tener por ejemplo dentro de nuestras habilidades de artesanías... o sea que para cada habilidad hubiera un taller [¿̨no?] adecuado... (C. Juajibioy, comunicación personal, 13 de julio de 2017).

Igualmente, la falta de recursos para la materia prima, pues para cada modalidad que trabaja la institución, se necesitan distintos materiales. Por ejemplo, en tejido en chaquira, la mostacilla como se conoce normalmente; en tejido en orlón, los orlones de lana, tablas y demás, los cuales no es deber de la institución hacerse cargo de todos los materiales. Los estudiantes, la mayoría provenientes de familias con escasos recursos económicos, son los más afectados por este problema, debido a que, en sus hogares, a veces no hay ni para la comida. La profesora Clara Juajibioy menciona que:

(...) lo otro es tener digamos, todos los recursos necesarios para poder desenvolverse dentro de... [ehhm] que los estudiantes tengan todo lo necesario, la materia prima para cada habilidad [ehh] es una dificultad que ellos tienen, porque pues siempre tienen que invertir [¿̨no?] los recursos por ejemplo en la compra de la chaquira, se exige que sea de calidad, la chaquira Checa. En Jajań, que todos vengan con la ropa adecuada, o sea venir con todo lo necesario, las herramientas adecuadas para poder trabajar en la chagra y bueno así en ese sentido existen esas dificultades (C. Juajibioy, comunicación personal, 13 de julio de 2017).

Además, la falta de recursos económicos para poder retribuir el apoyo brindado por las personas sabedoras de la comunidad (ancianos, mamitas, taitas, etc.) que desde los inicios han acompañado el proceso de formación del indígena Kamëntŝá, dando talleres de lengua materna, historia, artesanías y de todo lo que se trabaja y vive en la comunidad. Como menciona la docente Clara Juajibioy:

(...) otra sería en el sentido de lo que es lo propio [digamos] como tener los recursos para que nos podamos apoyar más en el conocimiento de nuestros abuelos, nuestros taitas para que ellos puedan [digamos] como que tuvieran un incentivo, o algo un recurso digamos económico para que nos puedan apoyar (C. Juajibioy, comunicación personal, 13 de julio de 2017).

Frente a esto la institución educativa, busca las formas de brindar el apoyo a los estudiantes y personas sabedoras de manera que el proceso de formación que han venido llevando desde hace más de 20 ańos, cada vez se fortalezca y se mantenga vivo por muchas generaciones.

8 Los docentes lo denominan "Taller de madera”, por ejemplo. 
Cuarta dificultad: a pesar de que existe el Proyecto Educativo Institucional (PEI), muchos docentes trabajan sus metas o sus objetivos educativos por fuera de éste, mas no proponiendo metas como una institución unida, que conlleven al cumplimiento de los objetivos trazados en el PEI como menciona el docente Miguel Mutumbajoy:

Una dificultad preocupante es la falta de organización por parte de toda la institución, porque para hacer una actividad en este sentido (organizar el PEI y demás actividades que requieren de la cooperación de toda la comunidad educativa) como que nos falta organización para que tengamos posibilidades. Cada uno hemos venido dependiendo de acuerdo a la preparación o investigación por cuenta propia que se hace, pero más no como institución, no nos hemos propuesto como metas institucionales, entonces no hemos podido avanzar... (M. Mutumbajoy, comunicación personal, 13 de julio de 2017).

Esta situación ha generado un estancamiento en cuanto a la construcción de nuevas propuestas educativas, en cooperación con la autoridad tradicional, padres de familia, y en general la comunidad educativa.

Pese a los factores que generan las grandes dificultades en la I.E. Bilingüe, padres, docentes y en general, la comunidad educativa, aunque sea en una cantidad no mayoritaria, en cuanto al desarrollo y cumplimiento a cabalidad de los fines establecidos en el proyecto educativo, buscan estrategias de intervención internas que fomenten y enriquezcan la diversidad de saberes existentes en la comunidad Kamëntŝ́á.

\section{Aprendizajes del proceso etnoeducativo}

Los docentes señalaron como aprendizajes significativos el haber contribuido en la formación educativa, cultural y personal de jóvenes, de los cuales, muchos siguieron cualificándose en habilidades de artesanías en sus diferentes modalidades, creando sus propios talleres artesanales y participando en diferentes eventos nacionales como menciona la docente Clara Juajibioy:

Digamos como ilustrativos de acá [¿̨no?]... de acá que ha habido jóvenes que han seguido [¿̨no?] digamos como cualificándose dentro de las artesanías y bueno se escucha que ya tienen digamos como formada su pequeña empresa o bueno han participado en algunas ferias (C. Juajibioy, comunicación personal, 13 de julio de 2017).

Así mismo, haber sembrado la semilla del fortalecimiento de la identidad cultural en cada uno de ellos, para facilitar los procesos de rescate en niños y jóvenes que vienen atrás.

Los docentes manifestaron una gran satisfacción en relación con que muchos estudiantes continúan estudios superiores en distintas universidades de Colombia en las diferentes ramas del conocimiento y regresan al territorio sin haber perdido el sentido de pertenencia hacia su comunidad y con nuevas ideas para continuar con 
la tarea del fortalecimiento de la identidad cultural de la comunidad Kamëntŝá, que les fue inculcada en la institución, como lo manifiesta la docente Clara Juajibioy:

\begin{abstract}
Los que han salido de la institución a estudiar a otras partes ya sea por el camino de las mismas habilidades artesanales (se refiere a un estudiante que estudió Artes Plásticas) y otros que han estudiado otras áreas del conocimiento han salido de aquí y han regresado ya profesionalizados y han vuelto hacia acá, al mismo pueblo, para contribuir y apoyar al colegio y a la comunidad... esa es una experiencia muy bonita y muy significativa para nosotros porque pues vemos que el trabajo no ha sido en vano [¿̨no?] (C. Juajibioy, comunicación personal, 13 de julio de 2017).
\end{abstract}

Además, algunos estudiantes egresados, continúan su proceso de fortalecimiento de la identidad cultural, siendo partícipes de los distintos espacios ofrecidos por la autoridad tradicional, por ejemplo, "hacen escuela", es decir, de forma gradual adquieren las habilidades para pertenecer al cabildo mayor, que es la máxima autoridad en la comunidad Kamëntŝá, convirtiéndose en líderes, comuneros y defensores del pueblo milenario, como la docente Clara Juajibioy dice:

Y dentro del fortalecimiento también [¿̨no?] han estado dentro de la presentación de servicio en el cabildo [¿no?], entonces han estado o han ejercido algunos como gobernadores. Para nosotros es agradable y a la vez pues o sea la institución ha sido de alguna forma motivadora en cuanto al proceso de cualificación que han tomado (C. Juajibioy, comunicación personal, 13 de julio de 2017).

El docente Miguel Mutumbajoy, manifiesta que como aprendizaje significativo de los docentes "en la parte cultural a uno le permite pensar, reflexionar, para aplicar ... uno ha tratado de buscar estrategias de aprendizaje para poder enseñar la lengua materna, entonces uno está investigando, con los mayores, aprende cada vez más" (M. Mutumbajoy, comunicación personal, 13 de julio de 2017). Pues para poder enseñar, cualquier asignatura, en este caso las áreas de la cultura, los docentes investigan y cada vez se apropian más de su cultura, visitan a los mayores sabedores y, por otro lado, desde la academia tradicional, buscan estrategias de enseñanza para lograr en los estudiantes incentivar el interés por aprender y rescatar los valores y saberes culturales.

\title{
5. Elementos etnomatemáticos presentes en el currículo etnoeducativo de la institución
}

Como ya se ha mencionado, la institución bilingüe establece en su Proyecto Educativo Institucional trabajar por el fortalecimiento de la identidad cultural de la comunidad Kamentsá, sin dejar de lado los conocimientos escolares establecidos por el Ministerio de Educación Nacional. En este sentido ha realizado esfuerzos en trabajar elementos culturales como la lengua, las plantas medicinales, la talla, la 
artesanía, pero en el área de matemáticas aún no es claro cómo se podría llevar la integración de los saberes matemáticos de la comunidad al aula de clase.

Para llevar a cabo dicha integración, es necesario iniciar por reconocer que el conocimiento matemático es una producción social que varía según el grupo sociocultural y el entorno en el que es generado, y por otra, que las matemáticas pueden contribuir a valorar y respetar la diversidad sociocultural de las personas (Peña-Rincón \& Blanco-Álvarez, 2015). De modo que, a través del diseño de propuestas de enseńanza, articulando los saberes ancestrales y occidentales, se enriquezca el currículo de matemáticas que la institución viene trabajando, brindando a los estudiantes herramientas que potencien el aprendizaje de la misma y sentido de pertenencia hacia su propia identidad.

Sin embargo, la integración de saberes etnomatemáticos se ha realizado de manera implícita, como se puede ver en la asignatura Artesanías, específicamente en el tejido Lana Orlón, se encuentra que dentro de estas prácticas autóctonas, como lo es el tejido del TŜ̀mbiach, en el que se plasma la simbología propia que dejaron los abuelos mayores, se promueven distintos tipos de pensamiento, geométrico, numérico y métrico, aunque no se tiene un fundamento teórico de éstos al estilo de la matemática escolar.

Para aprender a elaborar el tŝombiach o faja tradicional Kamëntŝá, se inicia elaborando el "diseño" de la figura que se va a plasmar, previo a una corta investigación a los abuelos sobre la importancia, el significado, las creencias, etc., que esta figura tiene para la comunidad. Cabe aclarar que cuando se dominan las figuras, no es necesario la elaboración del diseño, sino que, mentalmente los estudiantes las mantienen vivas.

En la figura 2, se ilustra el diseño del Sbaruk, en lengua materna o canasto en español, que es un objeto utilizado (hoy en día es poco común) para llevar los productos que se cultivan en el Jajañ o chagra. Para cada "renglón” está la descripción del paso para tejer, por ejemplo, sibiokan utataj traduce: "alza dos, deja dos, alza dos, deja dos, ...". Cabe resaltar que para el diseño de la "labor" (figura del canasto, parte central roja) se consideran los "hilos" por pares. Para el segundo renglón, Kanyaj juajames jatsbanam kantaj, kantaj juajames jatsbanam kantaj traduce: "deja uno, alza cuatro, deja cuatro y alza cuatro".

En el transcurso de la elaboración de la faja, el docente apoya a los estudiantes y comparte los conocimientos tradicionales que obtuvo de generaciones pasadas, haciendo de su clase "un entorno de aprendizaje propio". Es decir, mientras los estudiantes tejen, el docente, cuenta los relatos, creencias, etc., que sus antepasados le legaron. Aunque no necesariamente siempre es así, pues como se dijo anteriormente, previo a la elaboración del diseño de la figura, se hace una breve investigación, por esa razón los estudiantes también comparten lo que sus papás, abuelos, les comentaron. 


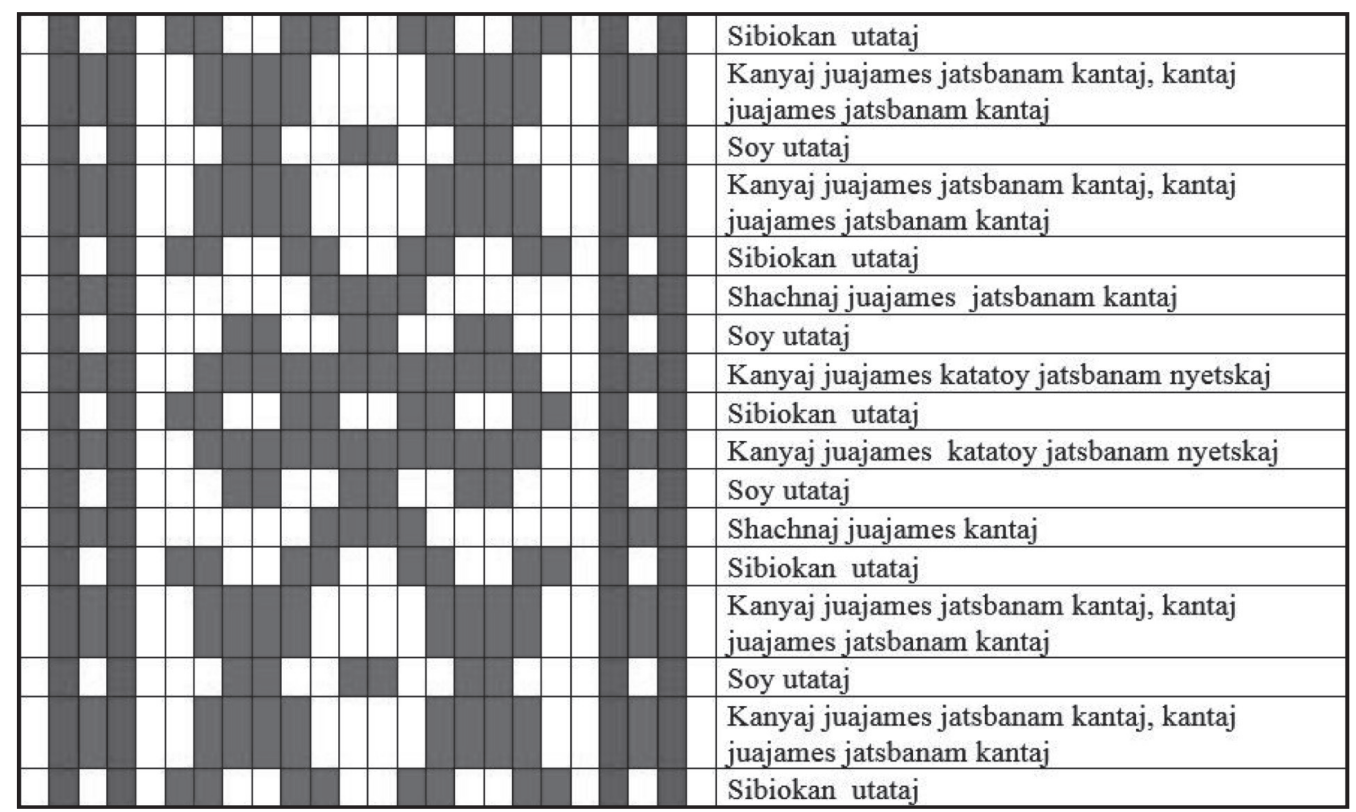

Figura 2. "Trabajo realizado por estudiantes del grado once en el año 2013 con acompañamiento de la docente Clementina Chicunque"

Fuente: propia

De otro lado, en la comunidad Kamëntŝá, existen diversas creencias, sobre el conteo, por ejemplo, los mayores dicen que contar las estrellas en la noche es malo, pues cuando muera, quien las cuenta, lo pondrán a contar harina.

En resumen, aunque existen ya elementos etnomatemáticos en el currículo, se hace necesario iniciar un proceso de indagación para responder a cuestiones como: ¿Cuál es el pensamiento geométrico de la comunidad Kamëntŝá, en particular, el que se trabaja en la institución educativa rural bilingüe artesanal Kamëntŝá? ¿Cómo diseñar actividades para el aula de clase desde una perspectiva etnomatemática, considerando los elementos culturales de los Kamëntŝá? La importancia del estudio de la etnomatemática en este caso, radica en brindar herramientas suficientes y necesarias que potencien el aprendizaje de las matemáticas y a la vez que fortalezcan los saberes de la cultura en los estudiantes del colegio bilingüe Kamëntŝá.

\section{Conclusiones}

Actualmente tanto la institución bilingüe como la misma comunidad Kamëntŝá, se enfrentan a un grave problema que bien podría enmarcarse dentro de lo lingüístico. El problema radica en que la mayoría de niños y jóvenes Kamëntŝá poseen mínimas competencias para la comprensión en lengua Kamëntŝá (entienden la lengua materna); mientras que la competencia para la producción es casi nula (no todos). Frente a esto, como se pudo evidenciar, en la institución bilingüe artesanal Kamën- 
tŝá, se trabajan los saberes propios desde las áreas de la cultura, establecidas en su proyecto educativo institucional a través de diferentes estrategias que confluyen hacia un mismo fin, la educación intercultural de los niños y jóvenes de la comunidad Kamëntŝá desde su misma cosmovisión y pensamiento ancestral.

En ese sentido, según Patiño (2004), la idea de que los centros educativos sean productivos, auto sostenibles e incluyan prácticas tradicionales de producción va calando poco a poco también en las comunidades indígenas. Es una manera de hacer interculturalidad en lo concreto y para beneficio general. Además, se puede considerar los estándares, o los currículos nacionales como una guía referencial para las instituciones educativas, sin embargo, como se mencionó anteriormente, gracias a la Ley General de Educación de 1994, las instituciones educativas, en este caso, la institución indígena bilingüe Kamëntŝá, tiene autonomía para establecer su horizonte institucional, de acuerdo a sus necesidades y cosmovisión propia. En ese sentido se puede hablar de la construcción de un proyecto educativo comunitario, donde no solo intervienen directivos, administrativos y docentes, sino también personas de la misma comunidad, el gobierno tradicional Kamëntŝá, y organizaciones que coadyuven en la pervivencia de los saberes tradicionales Kamëntŝá.

En particular, cabría pensar en que incluir las etnomatemáticas en el proyecto educativo comunitario, en el currículo y en el aula de clase, como plantean Peña-Rincón \& Blanco-Álvarez (2015), permite ampliar la comprensión de las matemáticas y hacer de la escuela un espacio incluyente y valorador de la diversidad sociocultural. Y tendría sentido hablar de una formalización de los saberes tradicionales presentes en las prácticas culturales de la comunidad Kamëntŝá, implementadas en el colegio bilingüe mediante la enseńanza de las áreas tradicionales.

En ese sentido (Peña-Rincon \& Blanco-Álvarez, 2015) señalan que la estructura didáctica que exponen (Johsua y Dupin, 1993) Saber Matemático-Estudiante-Maestro, los tres agentes en conexión, para trabajar la enseñanza y aprendizaje de las matemáticas desde la etnomatemática, es obsoleta, ésta debe enfrentarse a nuevos desafíos, entre los que groso modo se pueden mencionar: el saber se ve desafiado a reconocer la existencia de una amplia diversidad de pensamientos matemáticos en el mundo. El estudiante se ve desafiado a reconocer y valorar la pluriculturalidad en las matemáticas, y respetar de la diversidad de pensamientos matemáticos. El educador se ve desafiado a fortalecer la idea de maestro-investigador, es decir, un maestro que en su práctica docente sea sensible a las problemáticas presentadas en el aula de matemáticas, y a partir de la sistematización, el análisis y la discusión de estas con un grupo de colegas, a la luz de marcos teóricos de la educación matemática plantee soluciones y las socialice en encuentros.

En un sentido amplio, al enfocar los procesos de aprendizaje y de enseñanza de las matemáticas desde el enfoque cultural, se aporta al fortalecimiento del Proyecto Educativo Institucional de la institución educativa rural bilingüe artesanal Kamëntŝ́á 


\section{Referencias}

Bishop, A. (1999). Enculturación matemática. Madrid: Paidós Ibérica.

Colegio Bilingüe Artesanal Camëntsá. (2015). Proyecto educativo indígena Camëntsá. Sibundoy: Documento institucional.

Johsua, S. y Dupin, J. (1993). Introduction à la didactique des sciences et des matématiques. Paris, Presses Universitaires de France.

Ministerio del Interior \& Cabildo Indígena Camëntsá Biyá de Sibundoy. (2012). Diagnóstico Plan Salvaguarda Camëntsá.

Ministerio de Educación Nacional-MEN (1994a). Ley General de Educación 115. Bogotá: Imprenta Nacional de Colombia.

— . (1994b). Decreto 1860 de 3 de agosto de 1994. Santa Fe de Bogotá.

Oliveras, M. L., \& Blanco-Álvarez, H. (2016). Integración de las etnomatemáticas en el aula de matemáticas: posibilidades y limitaciones. BOLEMA: Boletim de Educação Matemática, 30(55), 455-480.

Patiño, E. (2004). Estado del arte de la etnoeducación en Colombia con énfasis en política pública. Bogotá: Ministerio de Educación Nacional.

Peinado, H. S., \& Rodríguez, J. H. (2007). Manual de gestión y administración educativa. Bogotá: Editorial Magisterio.

Peña-Rincón, P., \& Blanco-Álvarez, H. (2015). Reflexiones sobre cultura, currículo y etnomatemáticas. In K. de la Garza \& R. Cortina (Eds.). Educación, pueblos indigenas e interculturalidad en América Latina (pp. 213-246). Quito: Ediciones Abya-Yala. 